\title{
Development of Intersection Collision Avoidance Algorithm for B2V Safety Service
}

\author{
Min Baek ${ }^{1}$, Hwan Lee ${ }^{1}$, Hyojin Choi ${ }^{1}$ and Kyunbyoung Ko ${ }^{1,2}$ \\ ${ }^{1}$ Department of IT Convergence, \\ ${ }^{2}$ Department of Control and Instrumentation Engineering, \\ Korea National University of Transportation, 50 Daehak-ro, Chungju-si, \\ Chungbuk, 380-702 Korea \\ ${ }^{1}$ \{gjcltms,malvino13,odo\}@ut.ac.kr, ${ }^{2}$ Email kbko@ut.ac.kr
}

\begin{abstract}
In this paper, we proposed the intersection collision avoidance (ICA) algorithm for bicycle to vehicle $(B 2 V)$ safety service and analyzed its performance by using 'PreScan' and 'MATLAB'. As typical type accidents related with bicycles, we consider the bicycle to vehicle $(B 2 V)$ communication. The derived analytical approach can explain the condition and effect of bicycle collisions. Furthermore, proposed algorithm has been confirmed by simulations. Consequently, the ICA developing methodology is verified to be used as a general tool to reduce the cost and the time consumption required for commercialization of safety services under IEEE 802.11p/wireless access in vehicular environments (WAVE) wireless networks.
\end{abstract}

Keywords: ICA, B2V, PreScan, MATLAB, WAVE

\section{Introduction}

Recently, there has been increasing demand on vehicular communications (vehicle-tovehicle (V2V) and vehicle to infrastructure (V2I)), for various applications such as road safety, route planning and collision avoidance [1-3]. To realize these applications, cooperative-intelligent transportation system has being developed based on V2V and V2I communications. IEEE 802.11p/wireless access in vehicular environments (WAVE) was developed to support C-ITS based on V2V and V2I communications [4-6]. The modulation format of IEEE $802.11 \mathrm{p} / \mathrm{WAVE}$ is based on orthogonal frequency division multiplexing (OFDM)[7-10]. It has the structure of forward-error correction (FEC), preamble-sequences and comb pilot-symbol schemes. Moreover, IEEE 802.11p standard has the medium access scheme of carrier sensing multiple access with collision avoidance (CSMA/CA) [10-11]. The U.S. Federal Communication Commission (FCC) allocated $75 \mathrm{MHz}$ spectrum at $5.9 \mathrm{GHz}$ for $\mathrm{V} 2 \mathrm{~V}$ and V2I communications. The transmission rates can be provided as ranging from $3 \mathrm{Mbps}$ to $27 \mathrm{Mbps}$ with $10 \mathrm{MHz}$ bandwidth and the coverage is supported up to $1 \mathrm{~km}$ under a variety of scenarios, such as urban, suburban, rural and freeway, with relative vehicle velocities of up to $30 \mathrm{~m} / \mathrm{s}$ [12]. In parallel, based on IEEE 802.11p/WAVE, there have been researches dealing with collision avoidance under V2X communications [11, 13, 15].

In this paper, we develop the intersection collision avoidance (ICA) algorithm for safety service focusing bicycle accidents under WAVE networks. Notice that many car accidents have been related with bicycles and a bicycle driver can have more damages. In Korea, the mode-share of the bicycle increased form $1.2 \%$ at the year 2005 to $2.2 \%$ at the year 2010 [14-16]. In addition, the accidents related with bicycle have been proportional to the growth of cyclists. For traffic accidents associated with bicycle, the amount of increase is $7.9 \%$ per year and the death toll is about 300 [17]. At 2013, the number of bicycle accidents was 13,852 and the death toll was 282 [17]. Typical types of accident 
are intersection collision (55\%) and longitudinal collision (25\%) [17]. Based on this, we proposed ICA algorithm for bicycle to vehicle (B2V) safety service based on 'PreScan' and 'Matlab' and then, its performance is analyzed by simulation [18].

The remainder of this paper is organized as follows: Section 2 and 3 describe 'PreScan' overview and the proposed collision avoidance algorithms. The simulation results are presented in Section 4 and also concluding remarks are given in Section 5.

\section{Buildscenario}

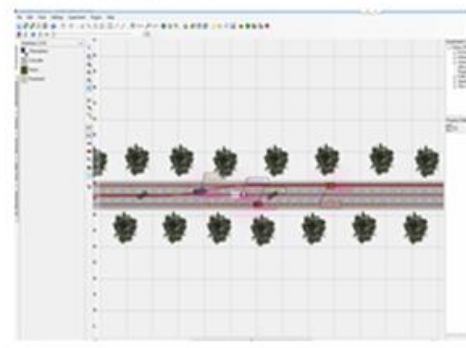

4. Run experiment

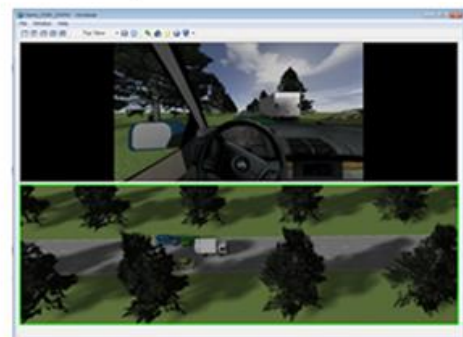

2. Model sensors
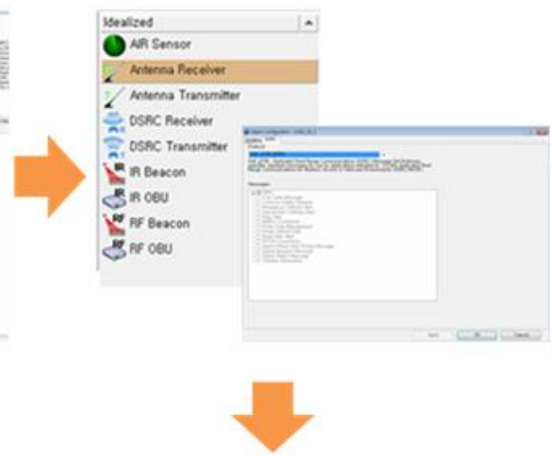

3.Add control system

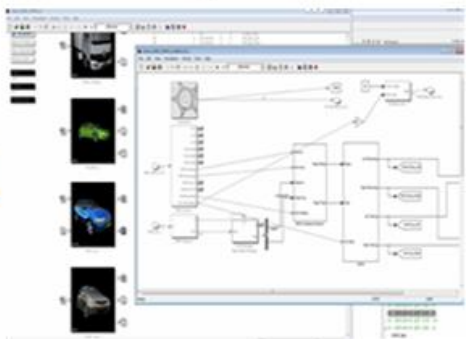

Figure 1. Developing Step by Prescan

\section{PreScan Overview [18]}

Note that 'PreScan' is a simulation platform consisting of a GUI-based preprocessor to define scenarios and a run-time environment to execute them [18]. The engineer's prime interface for making and testing algorithms includes 'MATLAB' and 'Simulink'. In addition, 'PreScan' can be utilized from modelbased controller design (MIL) to real-time tests with software in-the-loop (SIL) and hardware-in-the-loop (HIL) systems. 'PreScan' can operate in open-loop \& closedloop and offline \& online mode. It is an open software platform which has flexible interfaces to link to 3rd party vehicle dynamics model (e.g. CarSIM, dSPACE ASM, etc) and 3rd party HIL simulators/hardware (e.g. ETAS, dSPACE, Vector). Moreover, it comprises several modules that together provide everything an advanced driver assistance system (ADAS) system developer needs. An intuitive graphical user interface (GUI) allows users to build their scenario and model their sensors, while the 'Matlab/Simulink' interface enables us to add a control system. This interface can also be used to import existing 'Matlab/Simulink' models such as vehicle dynamics models. When running experiment, the visualization viewer gives a realistic 3D representation of the scenario. Optionally, tools such as dSPACE ControlDesk and NI LabVIEW can be used for activities such as 'data acquisition' and 'test automation'. The program works using four easy steps (see figure 1): 


\subsection{Build Scenario}

A dedicated pre-processor allows users to build and modify traffic scenarios within minutes using a database of road sections, infrastructure components (trees, buildings, traffic signs), actors (cars, trucks, bikes and pedestrians), weather conditions (such as rain, snow and fog) and light sources (such as the sun, headlights and lampposts). Representations of real roads can be quickly made by reading in information from OpenStreetMap, Google Earth, Google 3D Warehouse and/or a GPS navigation device [18].

\subsection{Model Sensors}

Vehicle models can be equipped with different sensor types, including radar, laser, camera, ultrasone, infrared, GPS and antennas for vehicle-to-X (V2X) communication. Sensor design and benchmarking is facilitated by easy exchange and modification of sensor type and sensor characteristics [18].

\subsection{Add Control System}

A Matlab/Simulink interface enables users to design and verify algorithms for data processing, sensor fusion, decision making and control as well as the re-use of existing Simulink models such as vehicle dynamics models from CarSim, Dyna4 or ASM [18].

\subsection{Run Experiment}

A 3D visualization viewer allows users to analyze the results of the experiment. It provides multiple viewpoints, intuitive navigation controls and picture and movie generation capabilities. Also, interfaces with ControlDesk and LabView can be used to automatically run an experiment batch of scenarios as well as to run hardware-inthe-loop (HIL) simulations [18].

\section{Development of ICA Algorithm for B2V Safety Service}

In this section, we proposed the safety service algorithm for B2V collision avoidance. For between vehicle and bicycle, let us consider WAVE communications and basic safety message (BSM) of the SAE J2735 Dedicated Short Range Communications (DSRC) Message Set Dictionary standard [19]. Note that the J2735 standard provides the foundation for a variety of applications including vehicle safety, emergency vehicle notification, automated tolling, enhanced navigation, traffic management and many others [19]. From the received BSM, a bicycle can know vehicle information ( GPS data, heading, yaw rate and so on). For a vehicle side, the bicycle's GPS data is only available. Under this constraint, we develop the ICA algorithm.

\subsection{Proposed ICA Algorithm}

Figure 2 and figure 3 show the ICA scenario and the proposed algorithm's block diagram for ICA, respectively [14]. From received two BSMs, a bicycle and a vehicle can calculate the velocities

$$
\begin{aligned}
& V_{B}=\rho_{B} \exp \left(j \theta_{B}\right)=\frac{D_{B}}{T_{M}}=\frac{P_{B N}-P_{B O}}{T_{M N}-T_{M O}} \\
& V_{V}=\rho_{V} \exp \left(j \theta_{V}\right)=\frac{D_{V}}{T_{M}}=\frac{P_{V N}-P_{V O}}{T_{M N}-T_{M O}},
\end{aligned}
$$




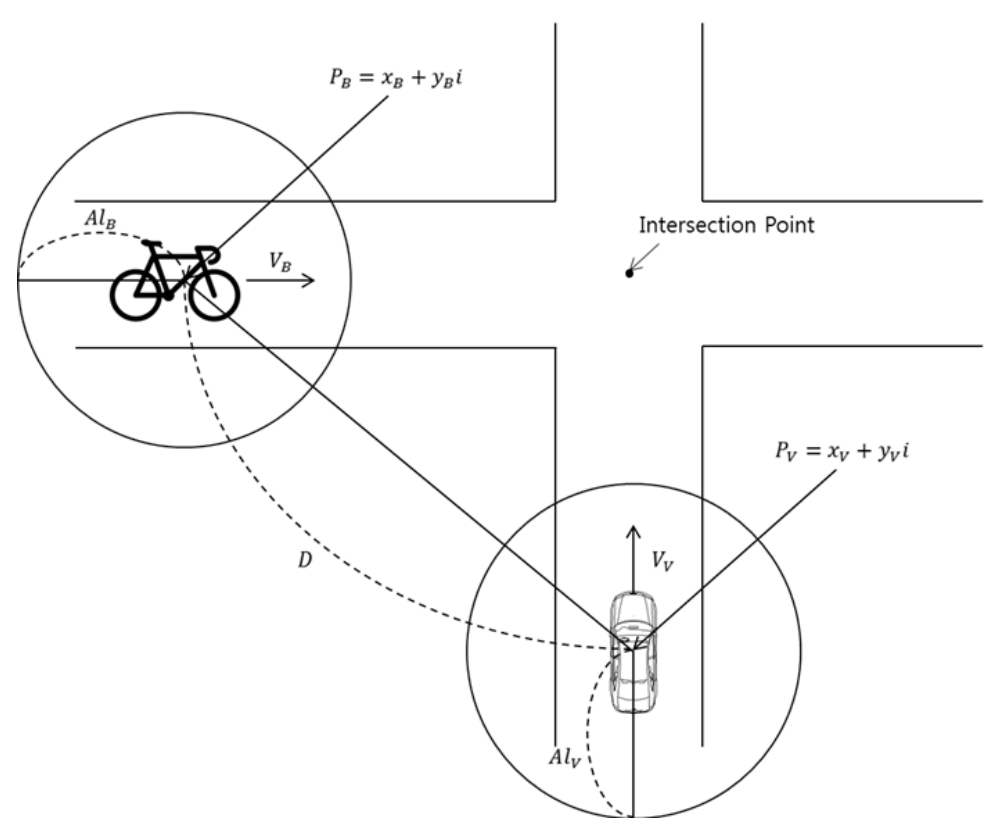

Figure 2. Intersection Collision Avoidance (ICA) Scenario

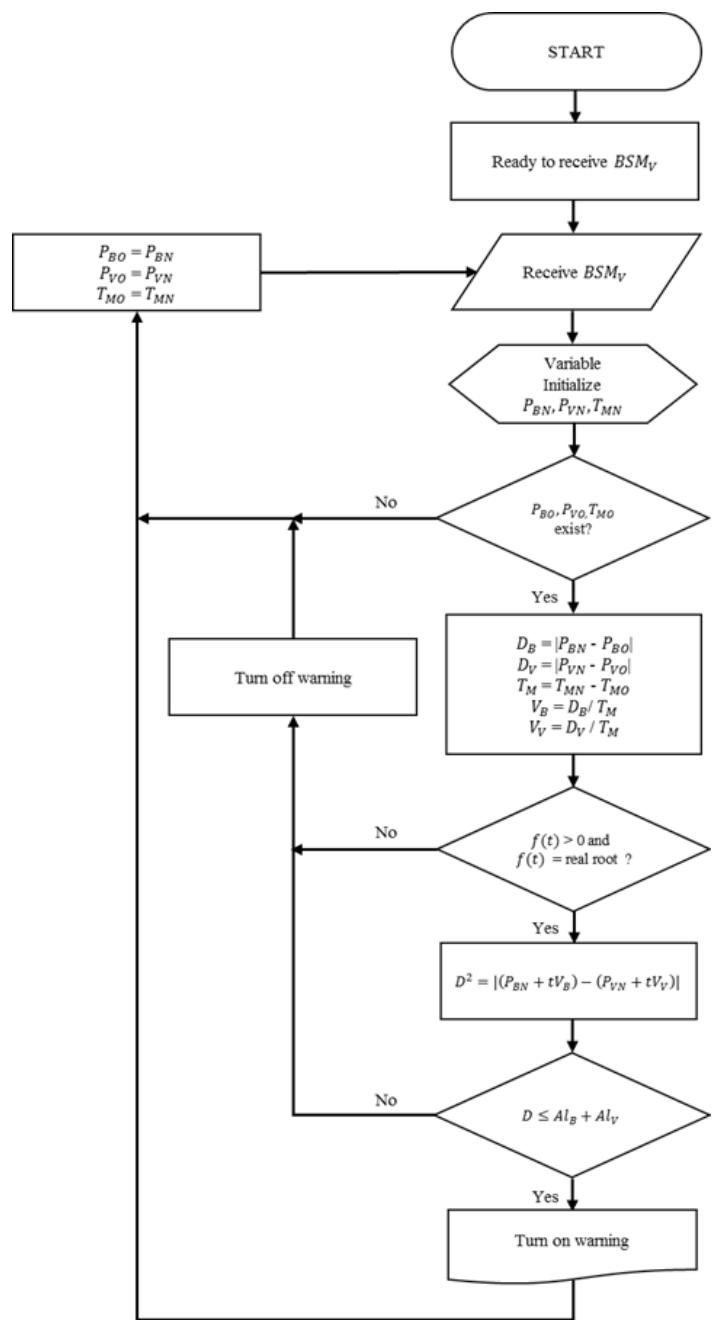

Figure 3. Flow Chart of Intersection Collision Avoidance (ICA) Algorithm 
where $T_{M}=T_{M N}-T_{M O}$ is the message time difference between two BSMs' receiving time ( $T_{M O}$ and $T_{M N}$ ). In addition, $D_{B}=P_{B N}-P_{B O}$ and $D_{V}=P_{V N}-P_{V O}$ are the distance between two GPS data. Note that subscript $B$ and $V$ indicate a bicycle and a vehicle and subscript $N$ and $O$ indicate new GPS data and old GPS data. After $t$ seconds, a bicycle and a vehicle can be located at $\left(P_{B N}+t V_{B}\right)$ and $\left(P_{V N}+t V_{V}\right)$, respectively. Then, the distance between bicycle and vehicle can be expressed as

$$
D^{2}=\left|\left(P_{B N}+t V_{B}\right)-\left(P_{V N}+t V_{V}\right)\right|^{2} \text {. }
$$

Note that as shown in figure 3, if $D \leq A I_{B}+A I_{V}$ is satisfied, a warning signal can be generated. By letting $D=A I_{B}+A I_{V}$ in (2), we can get a quadratic equation of

$A t^{2}+2 B t+C=0$

with

$$
\begin{aligned}
& A=|a|^{2}=\left|V_{V}-V_{B}\right|^{2} \\
& B=\operatorname{Real}\left\{(a)(b)^{*}\right\}=\operatorname{Real}\left\{\left(P_{B N}-P_{V N}\right)\left(V_{V}-V_{B}\right)^{*}\right\} \\
& C=|a|^{2}-\left(A I_{B}+A I_{V}\right)^{2} .
\end{aligned}
$$

Note that $A I_{B}$ and $A I_{V}$ are alram intervals of bicycle and vehicle as shown as Fig. 2, respectively. And then, we can obtain the solution of (4) as

$$
t=\frac{-B \pm \sqrt{B^{2}-A C}}{A}
$$

where $t$ of (5) maens time to collision (TTC). From (5), we can classify two cases for collision. At first case, if the solution of (5) has an imaginary root, there is no warning alram. It means that the distance $D$ of (2) cannot be less than $\left(A I_{B}+A I_{V}\right)$. The example for this (no collision or no warning) case is that after a vehicle passes through the intersection, a bicycle is approaching the intersection. On the other hand, if we have two real roots ( $t_{1}$ and $t_{2}$ with $\left.t_{1}<t_{2}\right), t_{1}$ is related with approaching and $t_{2}$ means the time from which the distance $D$ of (2) is larger than $\left(A I_{B}+A I_{V}\right)$.

\subsection{Proposed ICA Algorithm's Implementation by 'PreScan'}

In this paper, we implement the ICA scenario of Figure 2 by using 'PreScan'. Note that we follow four steps of Figure 1. At first, we build the ICA scenario of Figure 2 as Figure 9(a). After the second step of 'Model sensors' which is including BSM module, we add control systems for vehicle and bicycle. For the sake of convenience, the control system for bicycle is shown in Figure 4. Furthermore, we implement ICA algorithm of Figure 3 by 'Matlab/Simulink' as Figure 5. Note that the ICA algorithm of Figure 5 is included the ICA algorithm block in Figure 4. The result of 'Run experiment' will be presented at Section 4. 

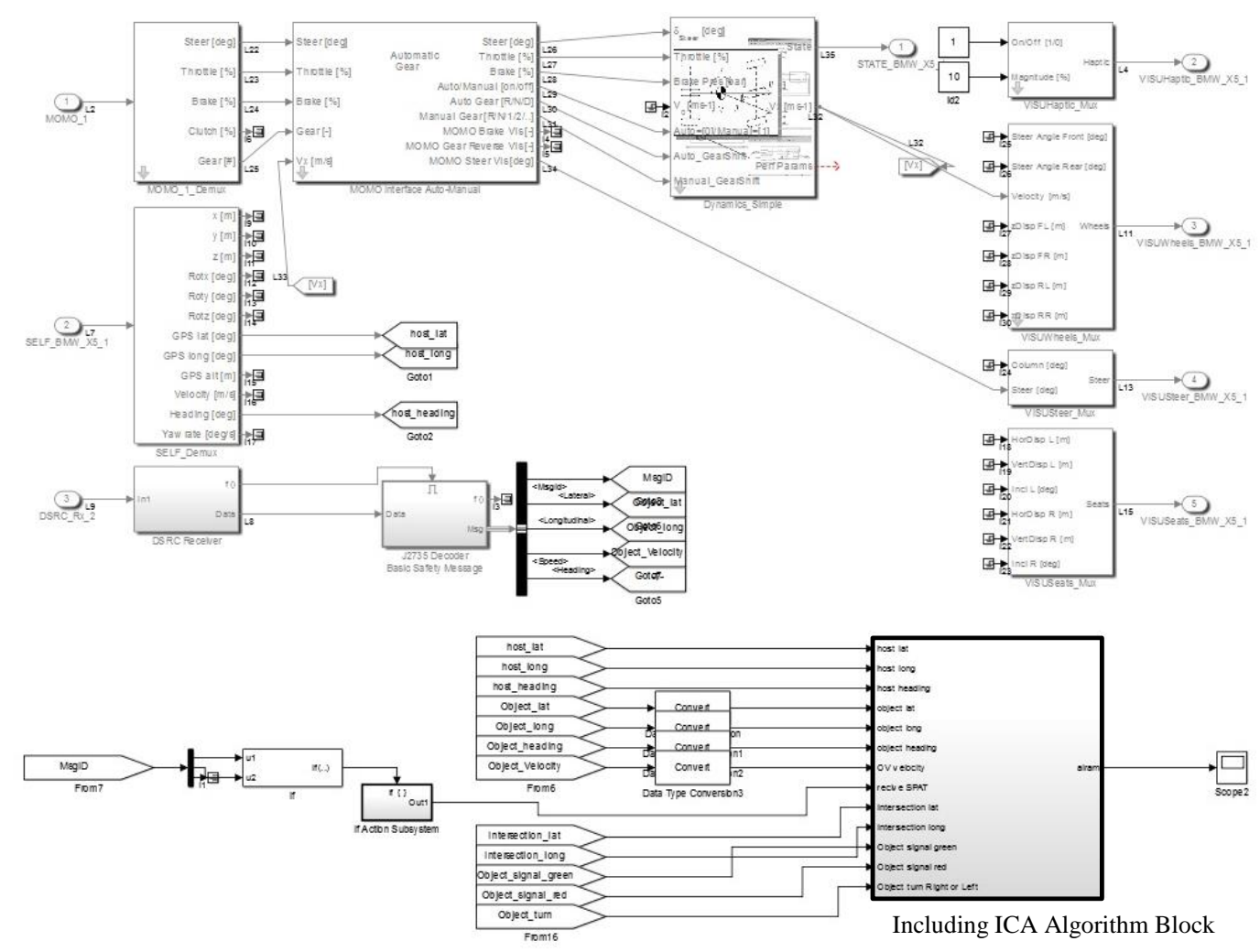

Figure 4. Control System for Bicycle

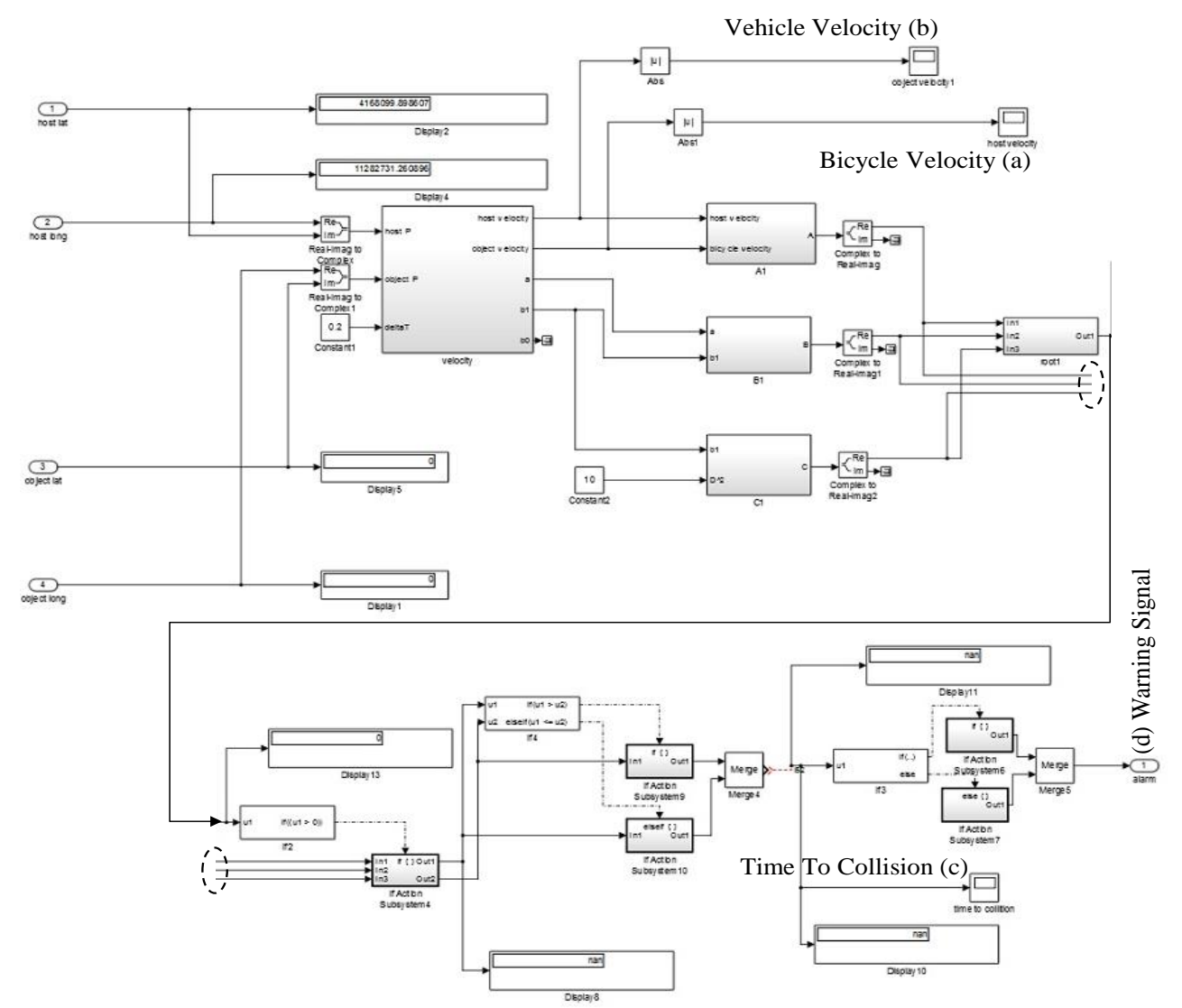

Figure 5. ICA Algorithm Implementation by Prescan 


\section{Table 1. Parameters of ICA Algorithm}

\begin{tabular}{|c|c|}
\hline $\begin{array}{c}\text { Parame } \\
\text { ter }\end{array}$ & Value \\
\hline $\begin{array}{c}V_{B} \\
m / s\end{array}$ & $5.5($ w/o deceleration $)$ \\
\cline { 2 - 3 } & $5.5 / 2(\mathrm{w} /$ deceleration $)$ \\
\hline $\begin{array}{c}V_{V} \\
m / s\end{array}$ & 11.0 or 20.0 \\
\hline$P_{B}(m)$ & $-160=(-160,0)$ \\
\hline$P_{V}(m)$ & $-j 300=(0,300)$ \\
\hline$\theta_{B}$ & 0 \\
\hline$\theta_{V}$ & $\pi / 2$ \\
\hline$A I_{B}$ & 5 \\
$(m)$ & \\
\hline$A I_{V}$ & 5 \\
$(m)$ & \\
\hline
\end{tabular}

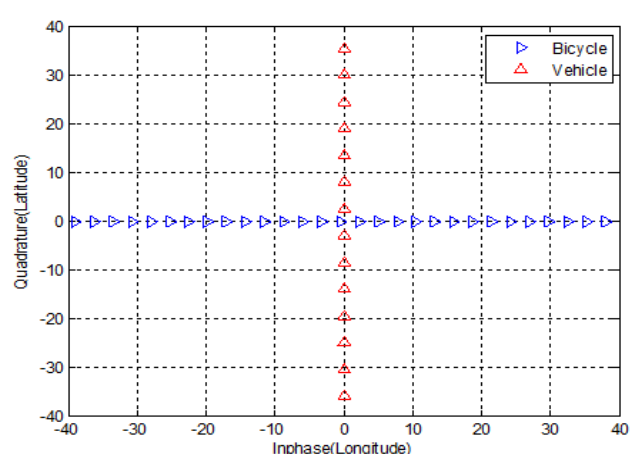

(A) Position

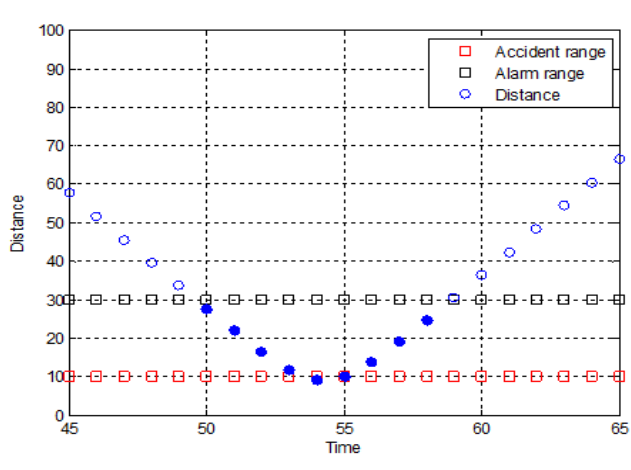

(B) Distance

Figure 6. Simulation Result of ICA Algorithm without Bicycle's Deceleration for Warning Signal $\left(V_{B}=5.5 \mathrm{~m} / \mathrm{s}, V_{V}=11 \mathrm{~m} / \mathrm{s}, A I_{B}+A I_{V}=30 \mathrm{~m}\right)$

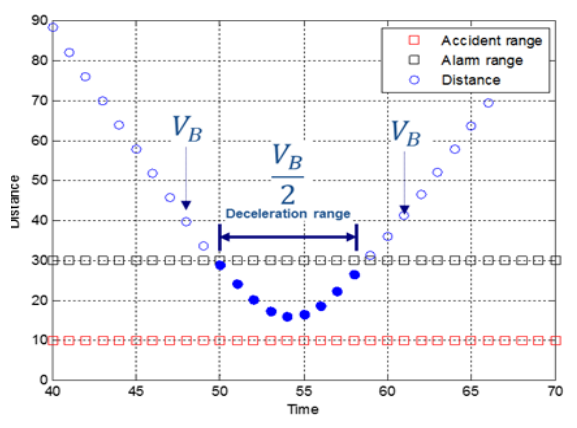

(a) Position

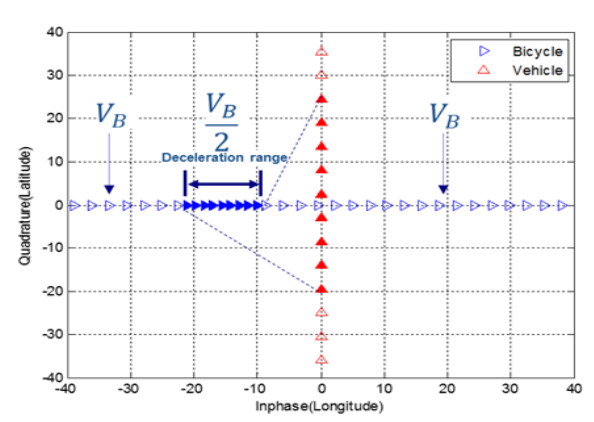

(B) Distance

Figure 7. Simulation Result of ICA Algorithm with Bicycle's Deceleration

For Warning Signal $\left(V_{B}=5.5 \mathrm{~m} / \mathrm{s}, V_{V}=20 \mathrm{~m} / \mathrm{s}, A I_{B}+A I_{V}=30 \mathrm{~m}\right)$ 


\section{Simulation Results}

In this paper, we utilize 'Matlab' and 'PreScan' in order to verify the functionality and the performance of the proposed ICA algorithm.

\subsection{Simulation Results by utilizing 'Matlab'}

Table 1 shows the simulation parameters of ICA algorithm. Using parameters of Table 1, we show the simulation results in Figure 6 and Figure 7. Figure 6 shows that the distance between bicycle and vehicle is less than $30 \mathrm{~m}$ from $t_{1}$ to $t_{2}$, which can be regard as a warning period. Note that in Figure 6, if two drivers ignore the warning signal, the distance can be less than $10 \mathrm{~m}$ and the collision cannot be avoided. On the contrary, in Figure 7, the bicycle driver decelerates as $V_{B} / 2$ within a warning period so that the distance cannot be less than $10 \mathrm{~m}$ and the collision can be avoided.

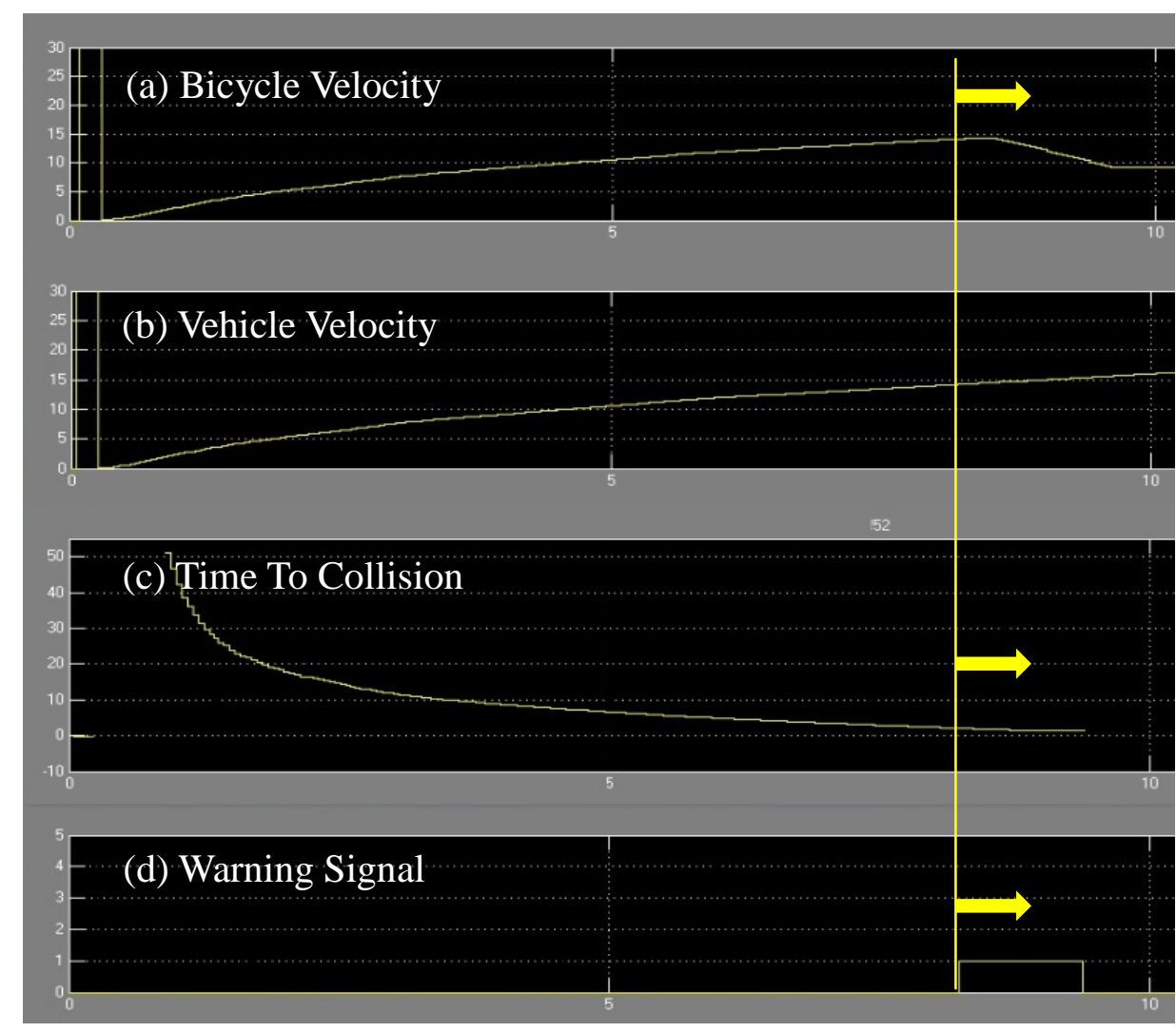

Figure 8. Simulation Result for Figure 5

\subsection{Simulation Results by utilizing 'PreScan'}

By utilizing the functionality of 'PreScan', the proposed algorithm is developed through 'Matlab/Simulink' interface as shown in Figure 1. In modeling process, we consider various environment with the different values for direction and velocity of both vehicle and bicycle (see Table 1).

Figure 8 and Figure 9 show simulation results of Figure 5 and scenes of run experiment, respectively. Figure 9(A) show the starting point of Figure 8 at time $=0$. As mentioned before, the proposed ICA algorithm in Figure 3 can can calculate TTC of (5) (see Figure 8(c)) and also generate the warning signal for the given condition (see Figure $8(\mathrm{~d})$ ). Then, the deriver can slow down the bicycle as shown 
Figure 8(a) and the vehicle goes without slowing down as shown Figure 8(b). This scene is shown as Figure 9(B). Therefore, the bicycle deriver can achieve the collision avoidance as shown in Figure $9(\mathrm{C})$.

\section{Conclusions}

In this paper, we proposed ICA algorithms based on B2V communication for bicycle safety services undeer IEEE $802.11 \mathrm{p} /$ WAVE wireless networks. The proposed algorithm is developed and analyzed by using 'PreScan' and 'Matlab'. Moreover, simulation results confirm its collision avoidance performance so that the developed methodology can be used as a tool to reduce the cost and the time consumption required for the realization of safety services based on B2V communications.

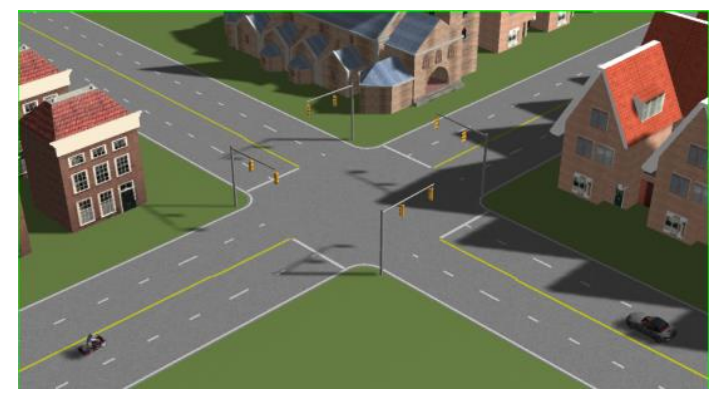

(A) Start

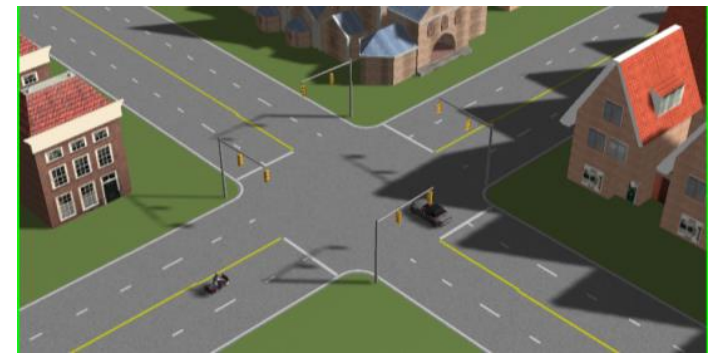

(B) Warning Signal

$\rightarrow$ Deceleration

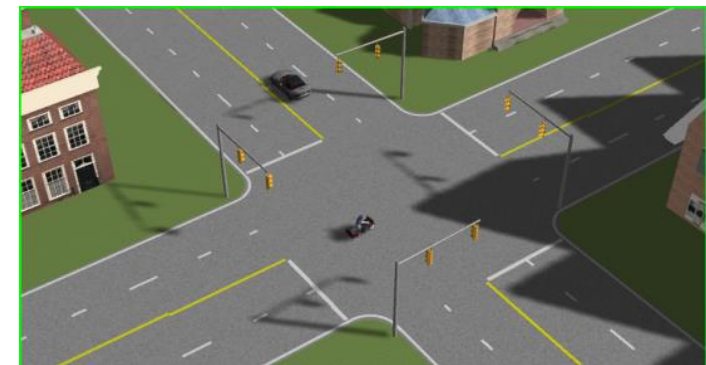

(C) Collision Avoidance

Figure 9. Run Experiment Result

\section{Acknowledgments}

This research was supported by a grant (14TLRP-C091025-01) from Transportation \& Logistics Research Program (TLRP) funded by Ministry of Land, Infrastructure and Transport of Korean government.

\section{References}

[1] F. Qu, F. Wang and L. Yang, "Intelligent transportation spaces: Vehicles, traffic, communications and beyond”, IEEE Commun. Mag., vol. 48, no. 11, (2010), pp. 136-142. 
[2] J. A. Fernandez, K. Borries, L. Cheng, B. V. K. Vijaya Kumar, D. D. Stancil and F. Bai, "Performance of the 802.11p Physical Layer in Vehicle-to-Vehicle Environments", IEEE Trans. Veh. Technol., vol. 61, no. 1, (2012), pp. 3-14.

[3] Zijun Zhao, Xiang Cheng, Miaowen Wen, Bingli Jiao and Cheng-Xiang Wang, "Channel Estimation Schemes for IEEE 802.11p Standard”, IEEE Intelligent Transportation Systems Mag., vol. 5, no. 4, (2013), pp. 38-49.

[4] IEEE802.11p, "IEEE Standard for Information Technology - Telecommunication and Information Exchange Between Systems - Local and Metropolitan Area Networks - Specific Requirements. part 11: Wireless LAN MAC and PHY Specifications - Amendment 6: Wireless Access in Vehicular Environments", http://www.ieee802.org/11/, (2010).

[5] Y.K. Kim, J.M. Oh, Y.H. Shin, C. Mun, "Time and Frequency Domain Channel Estimation Scheme for IEEE 802.11p", Proceeding of 2014 IEEE 17th International Conference on Intelligent Transportation Systems (ITSC), pp. 1085--1090, Qingdao, China., (2014), Oct. 8-11.

[6] G.W. Seo, M. Baek, C.H. Bong and K.B. Ko, "Performance Evaluation for Cooperative ADF Relaying V2I Communications with Burst transmission and PSA-CE schemes Over Quasi-Static Rayleigh Fading channels", Proceeding of 2014 IEEE 17th International Conference on Intelligent Transportation Systems (ITSC), pp. 2101--2106, Qingdao, China., (2014), Oct. 8-11.

[7] KyunByoung Ko, Myonghee Park and Daesik Hong, "Performance Analysis of Asynchronous MCCDMA systems with a Guard Period in the form of a Cyclic Prefix", IEEE Trans. Commun., vol. 54, no. 2, (2006), pp. 216-220.

[8] Myonghee Park, Kyunbyoung Ko, Byungjoon Park and Daesik Hong, "Effects of Asynchronous MAI on Average SEP Performance of OFDMA Uplink Systems over Frequency-Selective Rayleigh Fading Channels", IEEE Trans. Commun., vol. 58, no. 2, (2010), pp. 586-599.

[9] Kussum Bhagat and Jyoteesh Malhotra, "Performance Evaluation of Channel Estimation Techniques in OFDM based Mobile Wireless System", International Journal of Future Generation Communication and Networking, vol. 8, no. 3, (2015), pp. 53-60.

[10] Shafi Shahsavar Mirza and Khaled M. Almustafa, "Estimation of Packet Loss on MAC Layer in IEEE 802.11 Wireless Local Area Networks", International Journal of Future Generation Communication and Networking, vol. 8, no. 2, (2015), pp. 115-124.

[11] Y. Yuan, R. Lei and L. Xue, "Performance and Reliability Analysis of IEEE 802.11p Safety Communication in a Highway Environment", IEEE Trans. Veh. Technol., vol. 62, no. 9, (2013), pp. 4198-4212.

[12] C. Han, M. Dianati, R. Tafazolli, R. Kernchen and X. Shen, "Analytical Study of the IEEE 802.11p MAC Sublayer in Vehicular Networks", IEEE Trans. on Intelligent Transprotation Systems, vol. 13, no. 2, (2012), pp. 873-886.

[13] W. Fehr, T. Lusco, F. Perry, J. Marousek, B. A. Hamilton, G. Krueger and D. McNamara, "Southeast Michigan 2014 Test Bed project for connected vehicles: The next step toward deploying ITS", Proceeding of 2013 International Conference on Connected Vehicles and Expo (ICCVE), (2013), pp. 66-70.

[14] Yeona Kim, Jisu Ryu, Min Baek, Hwan Lee, Cheol Mun and Kyunbyoung Ko, "Development and Performance Analysis of Bicycle Collision Avoidance (BCA) Algorithms by Using PreScan and MATLAB", Proceeding of 2015 IEEE 18th International Conference on Intelligent Transportation Systems (ITSC), pp. 1506-1511, Canary Islands, Spain, (2015), Sept. 15-18.

[15] ROADSAFE, http://www.ftw.at/projects/roadsafe.

[16] Korea's Transport Database, http://www.ktdb.go.kr/en/web/guest/home.

[17] Traffic Accident Analysis System, https://taas.koroad.or.kr/Eng/indexMain.jsp.

[18] PreScan Overview, https://www.tassinternational.com/prescan-overview.

[19] ITS Standards Program, http://www.standards.its.dot.gov/Factsheets/Factsheet/71.

\section{Authors}

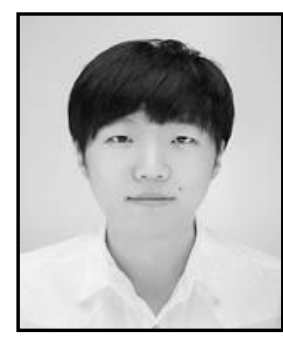

Hwan Lee, received the B.S. degrees in the Department of Control and Instrumentation Engineering at Korea National University of Transportation, Chungju, Korea in 2015. Currently, he is working toward the M.S. degree in the Department of IT Convergence at Korea National University of Transportation. His current research interests include the field of wireless communications focusing on CITS. 


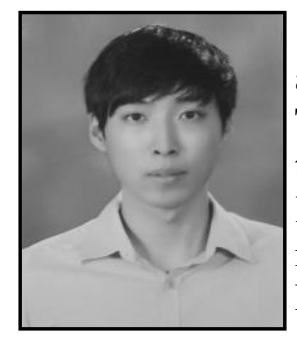

Min Baek, received the B.S. degrees in the Department of Control and Instrumentation Engineering at Korea National University of Transportation, Chungju, Korea in 2014. Currently, he is working toward the M.S. degree in the Department of IT Convergence at Korea National University of Transportation. His current research interests include the field of wireless communications focusing on CITS.

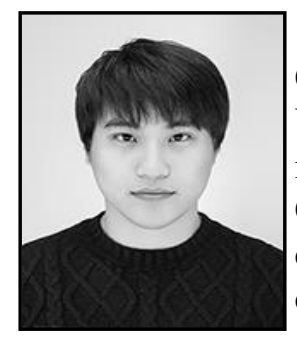

Hyojin Choi, received the B.S. degrees in the Department of Control and Instrumentation Engineering at Korea National University of Transportation, Chungju, Korea in 2015. Currently, he is working toward the M.S. degree in the Department of IT Convergence at Korea National University of Transportation. His current research interests include the field of wireless communications focusing on C-ITS.

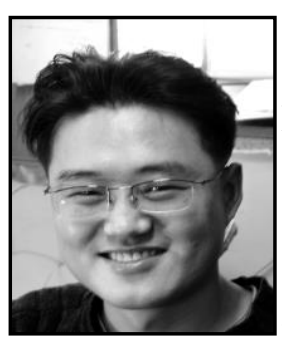

Kyunbyoung Ko, (Corresponding Author) was born in Seoul, Korea. He received the B.S., M.S. and Ph.D. degrees in Electrical and Electronic Engineering at Yonsei University, Seoul, Korea in 1997, 1999 and 2004, respectively. From March 2004 to February 2007, he was a senior engineer in Samsung Electronics Company, Ltd., Suwon, Korea where he developed Mobile WiMAX systems for broadband wireless services. He is currently an associate professor in the Department of Control and Instrumentation Engineering at Korea National University of Transportation. His current research interests include the field of wireless communications focusing on multicarrier and multi-antenna systems, cooperative relaying and C-ITS. 
International Journal of Control and Automation Vol.8, No.12 (2015) 